\title{
A non-anesthetized mouse model for recording sensory urinary bladder activity
}

\section{Peter Zvara ${ }^{\text {* }}$, Andrew J. Wright ${ }^{1}$, Kristopher Roach ${ }^{1}$, Michal Ursiny' , Bennett Shapiro ${ }^{1}$, Lawrence M. Dagrosa', Mark T. Nelson ${ }^{2}$ and Thomas J. Heppner ${ }^{2}$}

\author{
Department of Surgery, University of Vermont College of Medicine, Burlington, VT, USA \\ 2 Department of Pharmacology, University of Vermont College of Medicine, Burlington, VT, USA
}

Edited by:

Stuart Mazzone,

University of Queensland, Australia

Reviewed by:

Lu-Yuan Lee,

University of Kentucky, USA

Michael Ruggieri,

Temple University, USA

\section{*Correspondence:}

Peter Zvara, Department of Surgery, College of Medicine, University of Vermont, D319 Given Building, 89

Beaumont Avenue, Burlington, VT 05405, USA.

e-mail: peter.zvara@uvm.edu
The goal of this study was to develop an in vivo awake mouse model for extracellular bladder sensory nerve recording. A bipolar $125-\mu \mathrm{m}$ silver electrode was positioned under a single postganglionic bladder nerve. Efferent nerve signals were eliminated by tying off the postganglionic bladder nerve between the major pelvic ganglion and the recording electrode. Sensory nerve activity was measured in the conscious animals $48 \mathrm{~h}$ after surgery during continuous intravesical infusion of $0.9 \%$ saline/ $0.5 \%$ acetic acid followed by $0.5 \%$ acetic acid with capsazepine $(10 \mu \mathrm{M})$ at a rate of $0.75 \mathrm{ml} / \mathrm{h}$. Continuous infusion of $0.9 \% \mathrm{NaCl}$ led to a gradual increase in the frequency of sensory nerve firing that peaked upon reaching threshold pressure. Non-micturition contractions were observed in some animals during filling and other animals exhibited only minimal pressure fluctuations; both types of events were associated with a rise in sensory nerve activity. Intravesical infusion of $0.5 \%$ acetic acid reduced the intermicturition interval. This was associated with a 2.1-fold increase in bladder pressure during filling and a two-fold increase at both threshold and micturition pressures. Concurrent with these changes, sensory activity increased 2.8-fold during filling and 2.4-fold at threshold pressure. Subsequent intravesical infusion of capsazepine in $0.5 \%$ acetic acid reduced filling and threshold pressures by 21 and $31.2 \%$, respectively, and produced corresponding decreases of 36 and $23.4 \%$ in sensory nerve activity. The current study shows that multifiber sensory nerve recordings can be reproducibly obtained from conscious mice.

Keywords: urinary bladder, sensory nerves, conscious mouse

\section{INTRODUCTION}

The afferent limb of the micturition reflex presents an attractive target for pharmacological intervention in the treatment of bladder dysfunction, but the animal models currently available for testing potential therapeutic agents have limitations (Andersson, 2002). Two types of in vitro preparations have been used to study bladder sensory activity in response to mechanical and chemical stimulation: an en block bladder/pelvic nerve preparation (Sengupta and Gebhart, 1994; Rong et al., 2002) and flat-sheet bladder preparation (Zagorodnyuk et al., 2006). Studies employing these models have contributed significantly to defining the peripheral neuronal mechanisms involved in bladder sensation. Studies focusing on central neuroregulation of the micturition reflex during bladder filling and micturition require an intact micturition reflex pathway and can therefore only be conducted in vivo. Animal models used to date require the use of anesthesia, which exerts its effects by enhancing inhibitory and/or suppressing excitatory neural output, likely compromising nerve activity recordings (Habler et al., 1990; Hara and Harris, 2002; Malley and Vizzard, 2002).

To address these limitations, we developed a new method for recording sensory nerve activity in vivo in the conscious animal. We have adapted the ex vivo technique of multifiber sensory nerve recording to in situ recordings from the postganglionic bladder nerve in a conscious mouse. Bladder-specific afferent neural discharge and corresponding changes in intravesical pressure during bladder filling were recorded. The validity of this model was tested by recording extracellular bladder sensory nerve activity in healthy animals and after inducing bladder overactivity with $0.5 \%$ acetic acid. The functional contribution of C-fibers to the bladder sensory discharge during noxious/chemically induced bladder irritation was assessed using the TRPV1 receptor antagonist, capsazepine (Daly et al., 2007).

\section{MATERIALS AND METHODS}

Male 6- to 8-week-old BalbC mice (Charles River Laboratories, St Constant, QC, Canada) were maintained under standard laboratory conditions with free access to food and water. All animal use procedures were approved by the University of Vermont Institutional Animal Care and Use Committee, and appropriate measures were taken to minimize pain and discomfort of the animals.

After anesthetizing mice with isoflurane, a lower midline abdominal incision was made and PE-10 tubing $\left(\mathrm{BD}^{\circ}\right)$ with a heat-flared end was implanted into the dome of the urinary bladder. Postganglionic bladder nerves running on the lateral aspect of the prostate were identified (Figure 1A), and a single branch was bluntly dissected approximately midway between the major pelvic ganglion (MPG) and the bladder neck. A 0.125-mm diameter, bipolar Teflon-coated silver electrode (WPI, Sarasota, FL, USA, catalog number AGT0510) with hook-shaped poles was positioned under the single postganglionic nerve. Isolation from surrounding 


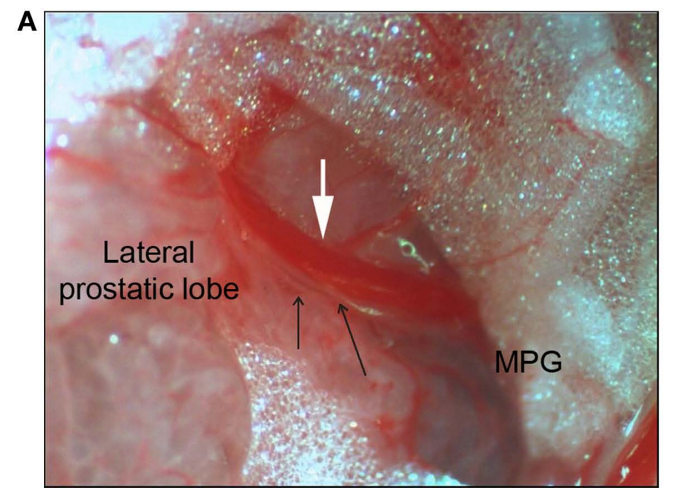

FIGURE 1 | (A) Postganglionic bladder nerves (two branches are marked by black arrows) running between the MPG and the bladder neck alongside the bladder vein (marked by white arrow) on the lateral aspect of the prostate (total magnification $\times 40$ ). (B) Depiction of the teflon-coated silver electrode with

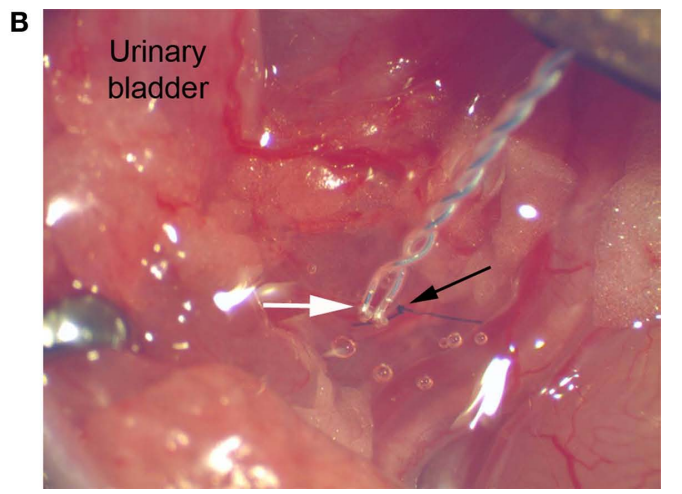

exposed hook-shaped poles lying beneath the postganglionic bladder nerve (marked by white arrow), embedded in the clear transparent silicone adhesive compound. Black arrow marks the 10-0 nylon ligature tied between the MPG and the recording electrode (total magnification $\times 30$ ). tissues was achieved by slightly lifting the nerve and the electrode of the prostatic surface using a micromanipulator Narishige MX-1 (Narishige Scientific Instrument Lab., Tokyo, Japan). After achieving a stable recording, the nerve was ligated between MPG and the electrode to eliminate efferent nerve signaling. The nerve-electrode complex was then embedded in a biocompatible silicone adhesive compound (KWIK-SIL; WPI, Sarasota, FL, USA; Figure 1B). This non-toxic compound secures the contact between the electrode and the nerve, anchors the nerve-electrode complex to the surrounding tissues, thereby preventing electrode movement and damage to the nerve. It also guarantees electrical isolation. The recording electrode and the ground reference electrode wire which was positioned within the abdominal cavity, were then externalized at the animal's back and secured to paravertebral muscles with 6-0 nylon sutures. The bladder tubing and the electrode were coiled into two separate subcutaneous pouches, and the animal was allowed to recover for $48 \mathrm{~h}$. Postoperative analgesia was maintained with buprenorphine ( $0.05 \mathrm{mg} / \mathrm{kg}$ s.c.). Analgesia was stopped $24 \mathrm{~h}$ prior to sensory nerve recording to prevent its suppressive effects on sensory neurotransmission.

The activity of sensory bladder nerves was recorded in situ in conscious animals. Under brief inhalation anesthesia with isoflurane, PE-10 tubing and the electrode and ground wire were removed from the subcutaneous pouches on the animal's back. The mouse was placed in a flat bottom mouse restrainer lined with an absorbent pad; the tubing and electrode were then connected to a pressure transducer (Grass Model PT300, Quincy, MA, USA) and a Neurolog head stage (NL100, Digitimer Ltd, UK), respectively. Intravesical pressure and sensory nerve activity were recorded simultaneously during continuous bladder filling. Intravesical pressure was recorded and analyzed using LabVIEW software (Texas Instruments, Dallas, TX, USA). Electrical activity was transmitted through the head stage, amplified (NL104), captured and analyzed via a power 1401 interface and Spike 2 software (version 6.03, CED, UK). Nerve activity was sampled at a rate of $25,000 \mathrm{~Hz}$ and stored on a PC. Off-line analysis was performed using the Spike 2 wavemark function; action potentials were discriminated based on their waveform.
The animal was allowed to acclimate 15-30 min before initiating continuous intravesical infusion of isotonic $0.9 \% \mathrm{NaCl}$ at a rate of $0.75 \mathrm{ml} / \mathrm{h}$. After three reproducible micturition cycles, the $\mathrm{NaCl}$ solution was replaced by $0.5 \%$ acetic acid (Sigma Chemical Co., St. Louis, MO, USA) diluted in $0.9 \%$ saline. In a subsequent set of experiments, $10 \mu \mathrm{M}$ capsazepine (Sigma) was added to the infusate after initial exposure to acetic acid. Intravesical pressure and sensory nerve activity were recorded simultaneously.

At the conclusion of the sensory nerve activity recording, the viability of the postganglionic bladder nerve was assessed. Neurostimulation, as square-wave pulses $(1.0 \mathrm{~ms}, 12 \mathrm{~Hz}, 4 \mathrm{~V})$, was applied through the bipolar silver electrode using a Grass stimulator (Grass Technologies, MA, USA). A segment of the postganglionic bladder nerve overlying the bipolar electrode was subsequently removed and evaluated histologically.

\section{DATA ANALYSIS}

The animal was continuously monitored and its physical activity was recorded. Artifacts in sensory recordings caused by movement were eliminated by analyzing only that data obtained during intervals when the animal was not moving. Whole-nerve multifiber afferent activity was expressed as impulses per second $(\mathrm{Hz})$ and is referred to as frequency of afferent nerve discharge. Data was quantified using a spike processor, which counts the number of action potential units per second that cross a preset threshold. The threshold value was set to the level of the smallest identifiable spike (approximately twice the baseline noise level). Nerve activity is expressed as a mean frequency of firing obtained over the course of $10 \mathrm{~s}$. Quantitative analyses compare data from three areas of interest within the micturition cycle before and after pharmacological intervention: (1) at the lowest pressure point during the filling phase (filling pressure), (2) at the voiding contraction threshold (threshold pressure), and (3) at peak micturition pressure. Quantitative values are presented as the mean \pm SEM, with differences between means determined using a paired Student's $t$-test. $P$-values $\leq 0.05$ were considered statistically significant. 


\section{RESULTS}

INTRAVESICAL PRESSURE DURING BLADDER FILLING AND MICTURITION

During continuous filling with $0.9 \% \mathrm{NaCl}$, cystometrograms showed two distinct patterns: (a) a steady intravesical pressure with pressure fluctuations of less than $2 \mathrm{~cm} \mathrm{H}_{2} \mathrm{O}$ (Figure 2A), and (b) a filling phase with pressure fluctuations ranging from 2 to $10 \mathrm{~cm} \mathrm{H}_{2} \mathrm{O}$ (Figure 2B).

Intravesical infusion of $0.5 \%$ acetic acid evoked a significant reduction in intermicturition intervals coupled with an increase in average filling and threshold pressures. The same mouse utilized for the basal and acetic acid urodynamic comparison was further challenged with a mixture of $0.5 \%$ acetic acid and $10 \mu \mathrm{M}$ capsazepine (Figure 3). In the presence of capsazepine, the bladder response to acetic acid was reduced as evidenced by an increase in intermicturition interval and a reduction in filling and threshold pressures.

\section{MULTIFIBER BLADDER SENSORY NERVE ACTIVITY DURING BLADDER FILLING}

Continuous filling with saline in vivo generated a reproducible pattern of afferent nerve discharge $(n=8)$. In all animals, baseline sensory activity oscillated between 20 and $60 \mathrm{~Hz}$, and repeated bursts of afferent discharge were observed throughout the entire filling phase (Figure 2). In some animals, low amplitude non-voiding bladder

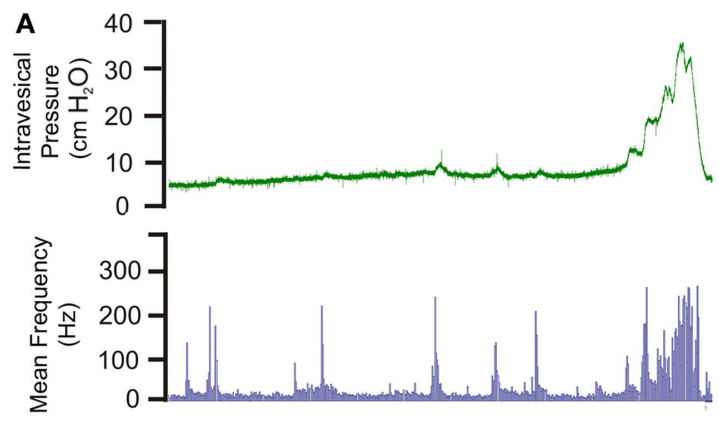

FIGURE 2 |Two distinct patterns recorded with cystometry: (A) Steady intravesical pressure during the filling phase with minimal pressure fluctuations; (B) Phasic components (small fluctuations in pressure) during the entire filling
B

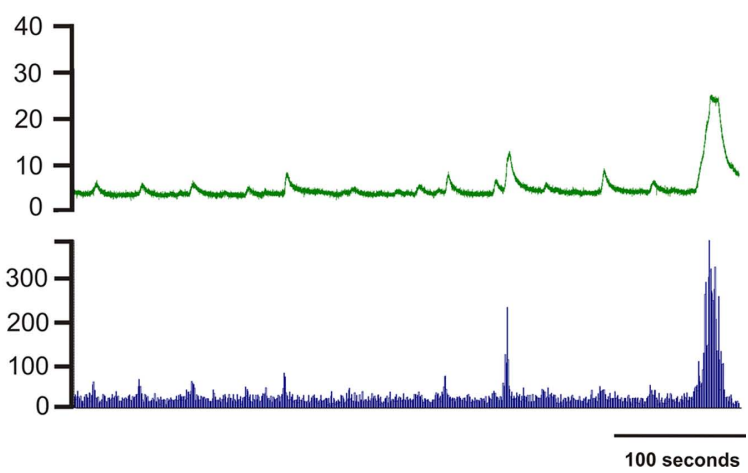

phase. The phasic intravesical pressure components were associated with bursts of afferent discharge. Phasic bursts of afferent activity were observed in all recordings regardless of pressure fluctuations.

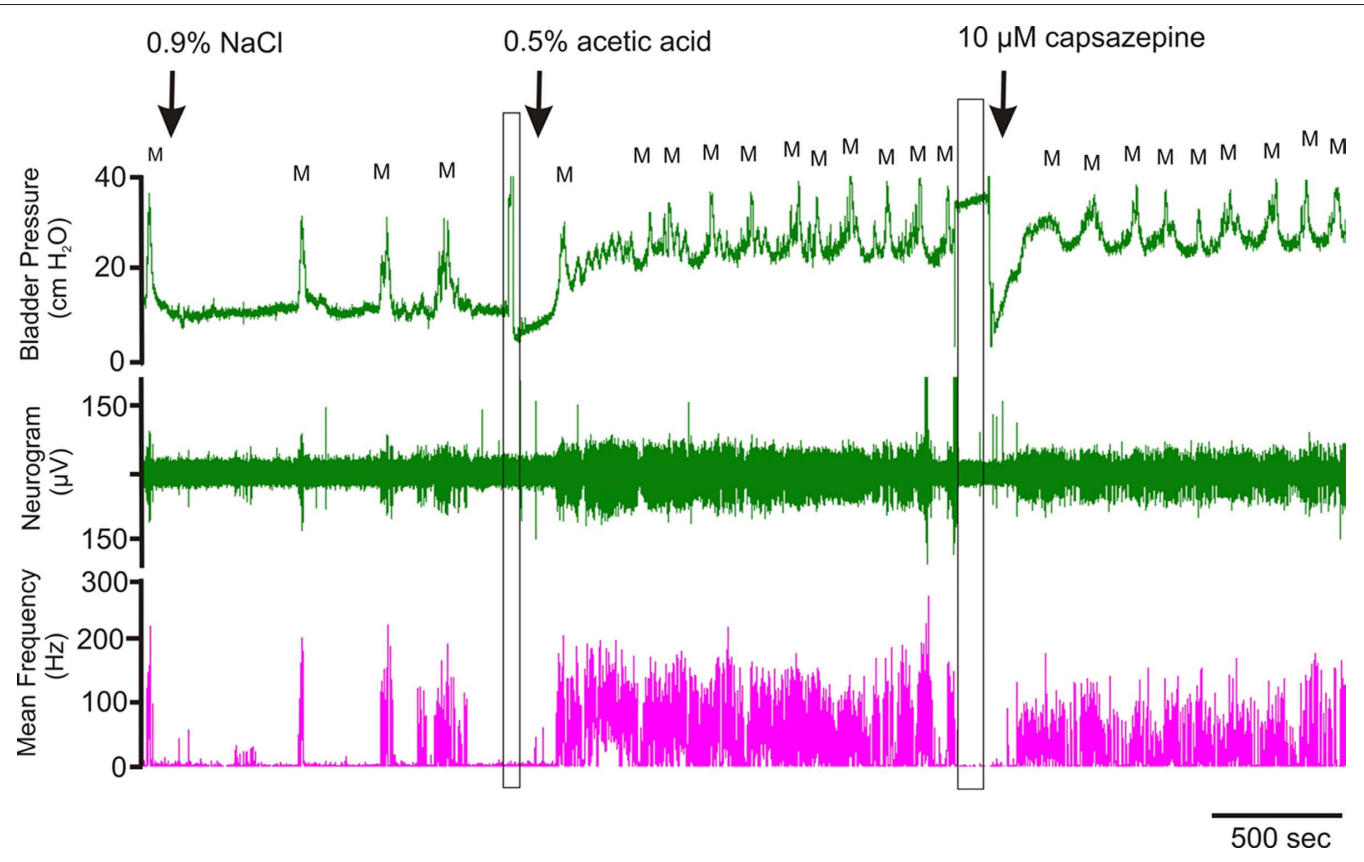

FIGURE 3 | Continuous recording of bladder pressure and afferent nerve activity. Infusion of $0.9 \% \mathrm{NaCl}$ led to regular micturition cycles with steady pressure during filling. $0.5 \%$ acetic acid led to higher micturition frequency, multiple non-voiding contractions along with elevated filling, threshold and micturition pressures. This change in bladder function was associated with increased nerve activity. Addition of capsazepine to the acetic acid solution reduced the number of non-voiding contractions and nerve activity. The rectangles mark the points in the experiment where the infusate was changed ( $M$, micturition event). 
contractions $\left(2-10 \mathrm{~cm} \mathrm{H}_{2} \mathrm{O}\right)$ seen throughout the filling phase correlated with phasic afferent discharge (Figure 2B). Phasic afferent discharge was also evident in a subgroup of animals that displayed only minimal fluctuations in intravesical pressure (amplitude $<2 \mathrm{~cm} \mathrm{H}_{2} \mathrm{O}$; Figure 2A). The terminal portion of the filling phase was characterized by a rapid increase in the frequency of sensory discharge that peaked at $97.4 \pm 20.0 \mathrm{~Hz}$ once the bladder reached the threshold pressure. The mean sensory nerve firing rate during micturition was $135.1 \pm 26.0 \mathrm{~Hz}$. In $20 \%$ of cases the experiment was unsuccessful due to excessive movement that lasted during entire recording.

Intravesical infusion of $0.5 \%$ acetic acid led to a 1.9 -fold increase in voiding frequency and reduced intermicturition intervals from $361.7 \pm 86.7$ s to $188.7 \pm 27.8 \mathrm{~s}(n=8)$. Acetic acid induced 2.1-fold, 2-fold, and 2-fold increases in filling, threshold, and micturition pressures, respectively, that were associated with 2.8-fold, 2.4-fold, and 1.9fold increases in excitatory afferent nerve discharge (Figure 4A).

Capsazepine opposed acetic acid-induced increases in the frequency of micturition and reduced bladder filling, threshold and micturition pressures by $25,31.2$, and $16.5 \%$ respectively $(n=8)$. The magnitude of the multifiber afferent response to bladder irritation with $0.5 \%$ acetic acid was significantly reduced in the presence of $10 \mu \mathrm{M}$ capsazepine. Acetic acid-evoked afferent discharge was inhibited by 36 and $23.4 \%$ at filling and threshold pressures, respectively, with no apparent effect on bladder contraction-related nerve activity (Figure 4B).

At the conclusion of sensory nerve recording, we evaluated the viability of the postganglionic nerve using electrical field stimulation. Electrostimulation-induced detrusor contraction resulted in an increase in intravesical pressure that ranged from 63 to $100 \%$ of the micturition pressure recorded during continuous bladder filling (Figure 5).

To address the potential impact of EMG artifacts originating from the striated urethral sphincter we conducted multifiber sensory recording with the nerve tied both proximal and distal to the recording electrode. Simply tying the nerve and elevating it of the surrounding tissues did not prevent the propagation the action potential. Insulating the nerve/electrode complex with silicone glue however resulted in complete signal cessation.

With the exception of microscopic foci of eosinophilic degenerative changes, morphological evaluation of the segment of the postganglionic bladder nerve overlying the electrode showed a normal morphology, consistent with a viable nerve. No inflammatory cells were present (Figure 6).

\section{DISCUSSION}

Functional properties of bladder afferent nerves have been studied extensively ex vivo. Mechano- and chemosensitivity have been recorded at the level of dorsal root afferent fibers in rats (Sengupta and Gebhart, 1994); pelvic nerves in mice (Rong et al., 2002; Daly et al., 2007), rats (Sengupta and Gebhart, 1994; Namasivayam et al., 1998), and cats (Floyd et al., 1976; Habler et al., 1990, 1993); lumbar splanchnic nerves in mice (Xu and Gebhart, 2008); and

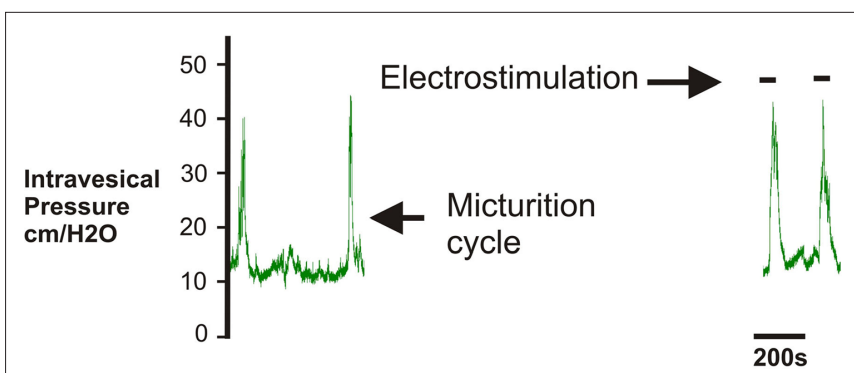

FIGURE 5 |At the conclusion of the experiment neurostimulation was applied to the postganglionic bladder nerve to confirm the viability of the nerve and specificity of the recording. Square-wave pulses $(0.3 \mathrm{~ms}$, $12 \mathrm{~Hz}, 2 \mathrm{~V}$ ) were delivered by a Grass stimulator, resulting in detrusor contraction with a rise in the intravesical pressure.

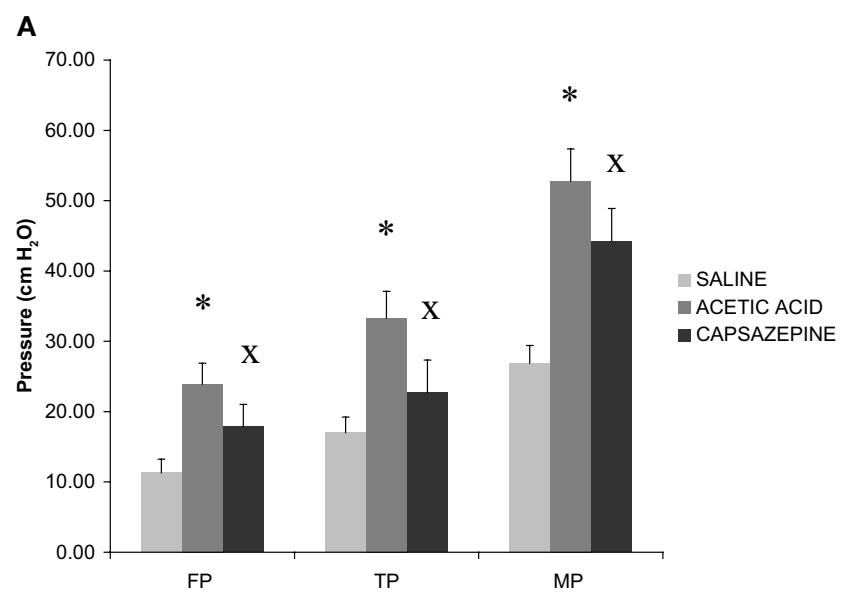

FIGURE 4 | (A) Barr graphs summarizing the data in filling (FP), threshold (TP), and micturition pressures (MP) $(n=8)$. A significant increase in all three parameters was observed following the infusion of $0.5 \%$ acetic acid. Capsazepine, infused with $0.5 \%$ acetic acid, significantly reduced filling and threshold pressures. The $\left(^{*}\right)$ mark a significant difference between $0.9 \% \mathrm{NaCl}$ and $0.5 \%$ acetic acid, ( $\left.{ }^{x}\right)$ indicate significant difference between $0.5 \%$ acetic acid and capsazepine. (B) Summary of

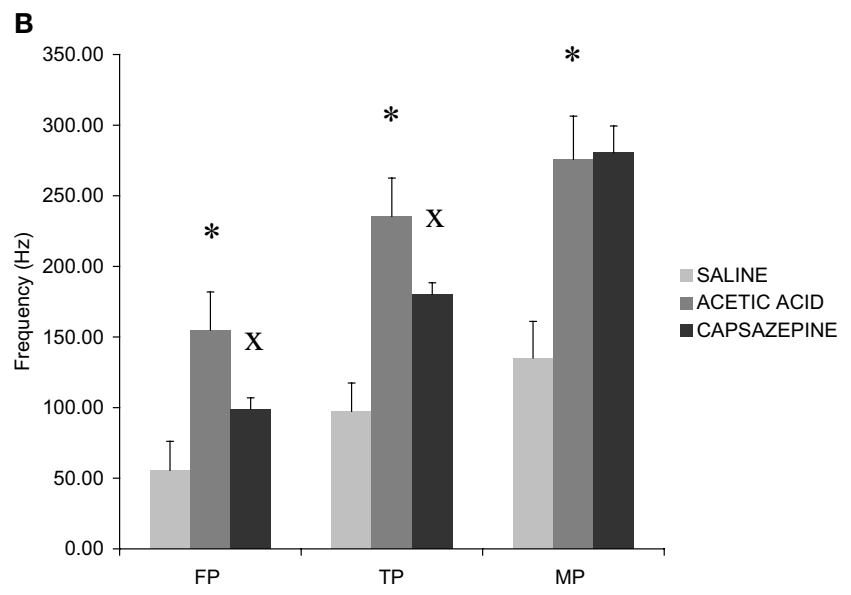

changes in sensory activity during filling, threshold, and micturition phases. A significant increase in all three parameters was observed following the infusion of $0.5 \%$ acetic acid. Capsazepine, infused with $0.5 \%$ acetic acid, significantly reduced sensory nerve activity recorded during filling and threshold phases. The $\left(^{*}\right)$ mark a significant difference between $0.9 \% \mathrm{NaCl}$ and $0.5 \%$ acetic acid, $\left({ }^{(}\right)$indicate significant difference between $0.5 \%$ acetic acid and capsazepine. 
A

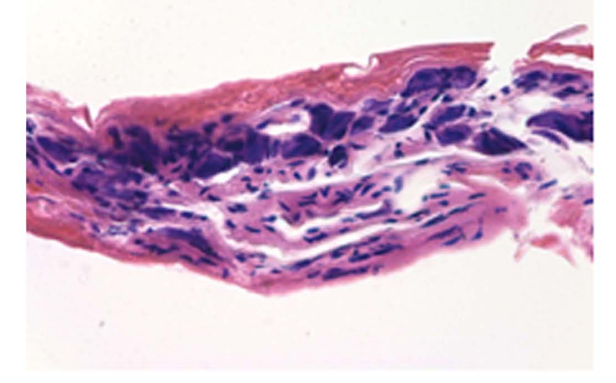

FIGURE 6 | Hematoxylin and eosin stain of postganglionic bladder nerves removed $48 \mathrm{~h}$ following implantation of the recording electrode. (A) The

section of the control nerve not affected by surgery. No inflammatory cells were
B

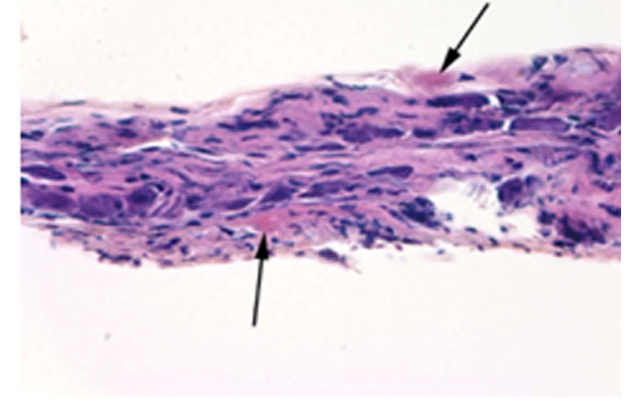

present. (B) The contralateral nerve that was overlying the electrode. The nerve was healthy except for two microscopic foci of eosinophilic degenerative changes (arrows). The total magnification $\times 400$. postganglionic bladder nerves in guinea pigs (Zagorodnyuk et al., 2006). In vivo anesthetized animal models (cats and rats) have been used as well (Mallory et al., 1989; Hara and Harris, 2002; Qin et al., 2003). In the present study, we introduce a new model for recording bladder-specific sensory nerve activity in vivo in a conscious mouse. The purpose of this model is to provide a new tool for the study of bladder sensory nerve activity that preserves micturition reflex pathways and avoids the suppressive effects of anesthesia on the nerve activity. This communication represents both a description and validation of the model.

In this study, continuous intravesical filling resulted in micturition cycles that were coupled with reproducible neural activity. During filling, sensory nerve activity recorded ex vivo was characterized by a gradual increase in frequency of multifiber nerve activity, whereas in vivo recording in a conscious mouse showed a complex pattern. In our study, a low level of relatively constant afferent nerve activity was observed during the initial phase of bladder filling. Small fluctuations in intravesical pressure $\left(<10 \mathrm{~cm} \mathrm{H}_{2} \mathrm{O}\right)$ that did not cause micturition led to bursts of afferent discharge. We were also able to detect bursts of afferent activity associated with minimal $\left(<2 \mathrm{~cm} \mathrm{H}_{2} \mathrm{O}\right)$ changes in the intravesical pressure. In this latter case, the observed sensory activity might have originated from micromotions in the bladder wall. Similar pressure transients have been reported in bladders of different animal species, including humans (Gillespie, 2004, 2005). In an ex vivo model, such spontaneous contractions have been recently shown to evoke afferent nerve firing in mouse bladders with detrusor overactivity (McCarthy et al., 2009). Our study provides further evidence that bursts of such sensory nerve activity likely play a role in the sensation of bladder filling in both health and disease.

In vivo models using anesthetized animals more faithfully mimic physiological conditions than do in vitro studies because they maintain the anatomical integrity of the micturition reflex. Urethane anesthesia, however, inhibits nociceptive neurotransmission through its effect on $N$-methyl-D-aspartate receptors (Hara and Harris, 2002). Mechanosensory and nociceptive responses to bladder filling in animal models of bladder overactivity may therefore be reduced by anesthetic suppression of signals transmitted by $\mathrm{C}$ - and/or A $\delta$ fibers. Urethane or chloral hydrate anesthesia also leads to less efficient voiding, as evidenced by the significant post-void residual volume and increased frequency of micturition (Streng et al., 2006).

To provide further insight into the contribution of different nerve populations, we used continuous intravesical infusions of $0.5 \%$ acetic acid to induce bladder overactivity. Acetic acid solutions have been shown to evoke a statistically significant increase in voiding frequency and intravesical pressure in conscious rats (McCafferty et al., 2008; Mitobe et al., 2008). Here we showed that acetic acid-induced changes are coupled with a higher frequency of sensory neural activity. We further documented reduced afferent responses to acetic acid in the presence of the TRPV1 receptor antagonist, capsazepine. Capsazepine reduced filling and threshold pressures by 36 and $23.4 \%$, respectively, providing additional support for a TRPV1-dependent mechanism in chemical bladder irritation. This finding was consistent with a recent report by Daly et al. (2007), which showed that the response to intravesical infusion of acetic acid was attenuated in ex vivo bladder preparations from anesthetized TRPV1 knockout mice. In our study, the sensory response to acetic acid infusion in a conscious mouse in the presence of capsazepine was reduced but not eliminated, suggesting that additional mechanisms are involved.

Mechanosensitive A delta fibers are low threshold fibers; therefore, it is possible that the increase in afferent firing and enhanced bladder activity was not due to direct excitation of afferents by acetic acid, but rather resulted from increased wall tension. A delta fibers, however, are connected to the detrusor muscle fibers in series (Gabella, 1995); thus, if they were the only fibers that contributed to the recorded sensory activity, the relationship between the bladder pressure and sensory firing would be linear. The correlation between the intravesical pressure and sensory nerve activity during bladder filling observed in our study was complex, and the increase in nerve discharge frequency during filling was non-linear. As evident from the data summary (Figure 4), changes in the frequency of afferent nerve discharge following intravesical infusion of acetic acid or capsazepine exceeded changes in the intravesical pressure. This suggests that afferent activity changes not only as a result of increased wall tension but also in response to direct stimulation of chemoreceptors and/or nociceptors in the bladder wall. 
To eliminate the efferent neurotransmission, we ligated the postganglionic bladder nerve between the electrode and MPG. The ligation likely has an effect on the nerve. The effect of ligation is likely similar to the process which takes place in the distal stump of the cut peripheral nerve. It was first described by Waller in 1850 and since then it has been confirmed by others (Stoll et al., 2002). "Wallerian degeneration" includes disintegration and degeneration of axoplasma and acrolemma. At the same time macrophage recruitment takes place, leading to rapid clearance of myelin debris, facilitating inflammatory processes leading to regeneration of the injured nerve. As shown by our experiments $48 \mathrm{~h}$ following ligation the nerve was viable. Electrostimulation of the postganglionic bladder nerve led to bladder contraction confirming preserved function. Hematoxylin and eosin staining showed that the morphology of the nerve overlying the electrode was preserved. Although eosinophilic degradation was evident, it was only present in the form of microscopic foci in the superficial portion of the nerve (Figure 5). In addition there is a possibility that ligation of the postganglionic bladder nerve might compromise bladder function. However, the fact that we have ligated only one of four to six terminal branches of this nerve entering each side of the bladder makes this unlikely. We compared our cystometry recordings to studies on healthy mice performed in our laboratory. This control comparison showed similar traces with comparable intravesical pressures during filling and micturition.

EMG signal is typically larger in magnitude than the nerve activity. Additionally, fluid and electrolytes in the abdominal cavity allow conduction. The potential impact of EMG artifacts was therefore

\section{REFERENCES}

Andersson, K.E. (2002). Bladder activation: afferent mechanisms. Urology 59,43-50.

Daly, D., Rong, W., Chess-Williams, R., Chapple, C., and Grundy, D. (2007). Bladder afferent sensitivity in wildtype and TRPV1 knockout mice. $J$. Physiol. 583, 663-674.

Floyd, K., Hick, V. E., and Morrison, J. F. (1976). Mechanosensitive afferent units in the hypogastric nerve of the cat. J. Physiol. 259, 457-471.

Gabella, G. (1995). The structural relations between nerve fibres and muscle cells in the urinary bladder of the rat. J. Neurocytol. 24, 159-187.

Gillespie, J. I. (2004). The autonomous bladder: a view of the origin of bladder overactivity and sensory urge. $B J U$ Int. 93, 478-483.

Gillespie,J.I. (2005).A developing view of the origins of urgency: theimportance of animalmodels. BJUInt. 96(Suppl.1),22-28.

Habler, H. J., Janig, W., and Koltzenburg, M. (1990).Activation of unmyelinated afferent fibres by mechanical stimuli and inflammation of the urinary bladder in the cat. J. Physiol. 425, 545-562.

Habler, H. J., Janig, W., and Koltzenburg, M. (1993). Receptive properties of myelinated primary afferents innervating the inflamed urinarybladder of the cat. J. Neurophysiol. 69, 395-405.

Hara, K., and Harris, R. A. (2002). The anesthetic mechanism of urethane: the

considered. We conducted multifiber sensory recording with the nerve tied both proximal and distal to the recording electrode. Simply tying the nerve and elevating it from the surrounding tissues did not prevent the propagation of nerve activity recording during bladder filling due to EMG artifacts. Imbedding the nerve/ electrode complex in the silicone glue however resulted in complete cessation of the signal, confirming that the activity recorded is solely attributed to the sensory nerve firing.

Our data provide evidence that an in vivo approach in a conscious animal yields reproducible results. Once we solved the technical issues and established the microsurgical techniques, only less than $20 \%$ of experiments had to be excluded from the analysis due to excessive movement artifacts. Preserved continuity of regulatory pathways and the absence of anesthesia allows for detailed study of afferent nerve function in health and disease. This model could complement currently available experimental methods in preclinical testing of therapeutic interventions that target the sensory limb of the micturition reflex. It is also applicable to genetically altered animal models, providing an opportunity to examine molecular targets relevant to bladder sensitivity and thereby adding a valuable tool for the study of fundamental bladder neurophysiology.

\section{ACKNOWLEDGMENT}

This study was supported by National Institute of Diabetes and Digestive and Kidney Disease Grants DK053832 and DK074173 to Mark Nelson and Peter Zvara respectively.

vitro bladder pelvic nerve afferent model in the rat. Br. J. Urol. 82 , 902-905.

Qin, C., Chandler, M. J., and Foreman, R. D. (2003). Effects of urinary bladder distension on activity of T3-T4 spinal neurons receiving cardiac and somatic noxious inputs in rats. Brain Res. 971 210-220.

Rong, W., Spyer, K. M., and Burnstock, G. (2002). Activation and sensitisation of low and high threshold afferent fibres mediated by $\mathrm{P} 2 \mathrm{X}$ receptors in the mouse urinarybladder. J. Physiol.541,591-600.

Sengupta, J. N., and Gebhart, G. F. (1994). Mechanosensitive properties of pelvic nerve afferent fibers innervating the urinary bladder of the rat. $J$. Neurophysiol. 72, 2420-2430.

Stoll, G., Jander, S., and Myers, R. R. (2002). Degeneration and regeneration of the peripheral nervous system: from Augustus Waller's observations to neuroinflammation. J. Peripher. Nerv. Syst. 7, 13-27.

Streng, T., Hedlund, P., Talo,A.,Andersson, K. E., and Gillespie, J. I. (2006). Phasic non-micturition contractions in the bladder of the anaesthetized and awake rat. BJU Int. 97, 1094-1101.

$\mathrm{Xu}$, L., and Gebhart, G. F. (2008) Characterization of mouse lumbar splanchnic and pelvic nerve urinary bladder mechanosensory afferents. $J$. Neurophysiol. 99, 244-253.
Zagorodnyuk, V. P., Costa, M., and Brookes, S. J. (2006). Major classes of sensory neurons to the urinary bladder. Auton. Neurosci. 126-127, 390-397.

Conflict of Interest Statement: The authors declare that the research was conducted in the absence of any commercial or financial relationships that could be construed as a potential conflict of interest.

Received: 28 April 2010; paper pending published: 13 May 2010; accepted: 02 September 2010; published online: 02 November 2010.

Citation: Zvara P, Wright AJ, Roach K, Ursiny M, Shapiro B, Dagrosa LM, Nelson MT and Heppner TJ (2010) A nonanesthetized mouse model for recording sensory urinary bladder activity. Front. Neur. 1:127. doi: 10.3389/fneur.2010.00127

This article was submitted to Frontiers in Autonomic Neuroscience, a specialty of Frontiers in Neurology.

Copyright (c) 2010 Zvara, Wright, Roach, Ursiny, Shapiro, Dagrosa, Nelson and Heppner. This is an open-access article subject to an exclusive license agreement between the authors and the Frontiers Research Foundation, which permits unrestricted use, distribution, and reproduction in any medium, provided the original authors and source are credited. 